\title{
Identification and Management of Head and Neck Cancer Recurrences Detected on Adjuvant Radiation Oncology Simulation Scans
}

\author{
RICHARD L. BAKST ${ }^{1}$ and WILLIAM SU ${ }^{2}$ \\ ${ }^{1}$ Department of Radiation Oncology, Mount Sinai Beth Israel, New York, NY, U.S.A.; \\ ${ }^{2}$ Icahn School of Medicine at Mount Sinai, New York, NY, U.S.A.
}

\begin{abstract}
Background/Aim: Computerized tomographic (CT) simulation is used to design precise radiation therapy treatment plans. These scans are not routinely read by radiology and are only used for planning purposes. As radiation oncology departments adopt the addition of intravenous contrast to their CT simulations, there is the potential for identification of post-surgical recurrences. Patients and Methods: We identified all biopsy-proven local recurrences of head and neck cancer detected during $C T$ simulation and followed their outcomes. Results: A total of 218 patients with head and neck cancer underwent CT simulation with intravenous contrast since our Department adopted the new technique. There were a total of four recurrences detected during simulation: two of the right tongue, one oral cavity and one laryngeal. Intensified salvage chemoradiotherapy was used for these cases. Two out of the four were successfully salvaged and remain disease-free. Conclusion: Recurrences of head and neck cancer following surgical resection can be detected on simulation scans, with the potential for survival after appropriate salvage therapy.
\end{abstract}

Late-stage head and neck cancer is typically managed in a multidisciplinary manner consisting of primary surgical resection followed by adjuvant radiotherapy (RT) or chemoradiotherapy (CRT) (1-5). Historically, simulations were completed in Radiation Oncology Departments without the use of intravenous (i.v.) contrast. These scans are not typically reviewed by radiologists. However, as more departments adopt the use of $i . v$. contrast for improved

Correspondence to: William Su, Icahn School of Medicine at Mount Sinai, One Gustave L. Levy Pl., New York, NY 10029, U.S.A. E-mail: William.Su@icahn.mssm.edu

Key Words: Recurrence, radiation, simulation, head and neck cancer, salvage therapy. planning techniques, an unintended consequence is the detection of postsurgical recurrences. Appropriate identification of failures is important in order not to underdose or undertreat gross disease. We reviewed our institutional experience since initiating the use of $i . v$. contrast with our computerized tomographic (CT) simulations.

At our Institution, we have detected select, aggressively returning head and neck cancer recurrences on CT imaging intended for simulation. Upon further evaluation, these patients were deemed to have unresectable recurrences. Thus, treatment for these patients was modified from adjuvant RT or CRT to salvage definitive CRT. CRT has been shown to be more effective for salvage treatment compared to RT alone for unresectable disease $(6,7)$. However, there is little information on how effective CRT is for those who experience quick recurrence after surgery and before initiation of adjuvant therapy. Herein, we report on salvage treatments used and the outcomes of these patients with aggressively recurring disease.

\section{Patients and Methods}

After receiving approval from the Institutional Review Board (GCO \#14-1075), all patients with head and neck cancer treated with primary surgical resection and planned for adjuvant therapy within our Radiation Oncology Department were identified. Those with biopsy-proven recurrence discovered during CT simulation with i.v. contrast were included. Furthermore, the included patients were not candidates for further surgical resection and, as a result, received salvage definitive CRT. A total of four patients were identified. Clinical documentation and electronic medical records were reviewed to determine how they were salvaged, their responses to treatment, and post-treatment outcomes.

CT simulation with i.v. contrast. Over a 3-year period, a total of 218 patients underwent CT simulation with i.v. contrast for their head and neck cancer at our Radiation Oncology Department. All included patients had gross total resection during surgery and those who experienced either distant or local recurrence during CT had biopsy-proven disease. 


\section{Results}

Patient characteristics. Four patients had local recurrence identified during CT simulation for planned adjuvant RT. Examples of initial imaging in which recurrence was originally suspected are shown in Figure 1. After identification, their recurrences were biopsy proven. All patients had undergone surgical resection of their primary tumor and were determined to be human papillomavirusnegative from pathology reports. Two patients had had primary tumors in the right tongue, another had had laryngeal cancer and the remaining patient had an oral cavity cancer. Three patients had significant positive smoking histories. Further patient details are shown in Table I.

Initial treatment plan. Initial adjuvant CRT or RT plans prior to detection of recurrence are shown in Table II. The RT dose initially planned was a median of $66 \mathrm{~Gy}$ (range=60-66 Gy).

Salvage treatment. All CT scans were reviewed with the surgeon and recurrences were deemed to be unresectable. Accordingly, patients were changed from a course of adjuvant RT or CRT to definitive CRT. All patients were salvaged with a total of 70 Gy of radiation along with concurrent weekly cisplatin. One of the patients, who had progressive disease despite initial salvage, underwent salvage with a combination of cisplatin, erbitux and 5-flurouracil afterwards. This was ineffective, and the patient is currently being treated with palliative erbitux. Detailed salvage therapy regimens are shown in Table II.

Outcomes. Two of the four total patients are currently alive with no evidence of disease 15 and 18 months after they completed salvage therapy. Another patient is alive 9 months after initial salvage treatment but has an aggressive buccal cancer recurrence and is receiving palliative chemotherapy. The remaining patient died 1 month after initial salvage treatment secondary to a pulmonary embolism.

Sample case report. A 52-year-old female with a significant 30 pack-year smoking history presented with stage III T3N1M0 squamous cell carcinoma of the larynx to our institution. She underwent a total laryngectomy. Surgical pathology revealed transglottic squamous cell carcinoma $(4.7 \mathrm{~cm})$ with 1 out of 74 lymph nodes being positive without extracapsular extension, no perineural invasion and no lymphovascular space invasion. Recurrence was found 6 weeks after the operation on CT simulation for RT, and was deemed to be unresectable. The superior soft-issue margin adjacent to the epiglottis was found to be positive for disease. As a result, the patient completed definitive concurrent CRT at 70 Gy (35 fractions of 2 Gy). At a followup time of 18 months after salvage treatment, the patient is

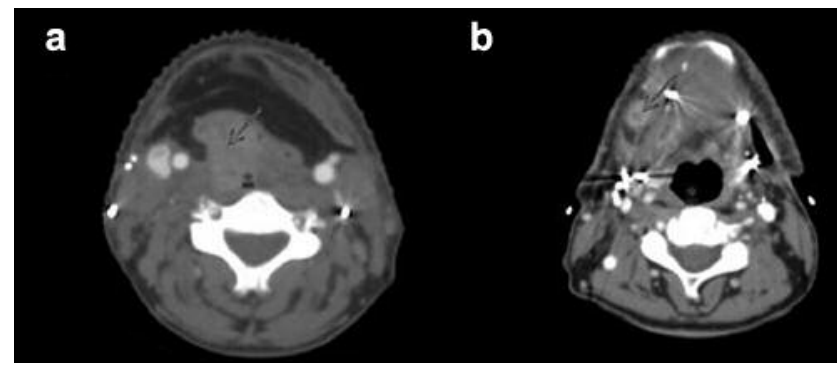

Figure 1. Detection of recurrence of head and neck cancer as shown on computed tomographic simulation with i.v. contrast. a: Recurrence at the surgical flap detected in a patient with primary laryngeal squamous cell carcinoma. b: Right level IB lymph node recurrence in a patient with a primary squamous cell carcinoma of the right tongue.

doing well with no significant complaints, being monitored with positron-emission tomography-CT surveillance and has no evidence of disease.

\section{Discussion}

This study suggests that local head and neck cancer recurrences may be detected if Radiation Oncology Departments that adopt the use of i.v. contrast as part of their simulation scan carefully observe CT simulation imaging. The recent addition of contrast agent during CT simulations has allowed for better visualization of structures and possible recurrences in patients. While not its original intention, CT simulation allows for an additional check-point at which patient disease status can be assessed. At our institution, CT simulations are used within the Radiation Oncology Department for treatment planning and are not read by diagnostic radiologists, unless the radiation oncologist suspects a recurrence and consults with one. All proceeding findings for patients should be reviewed with radiologists. Despite the quick recurrence that patients in our study experienced after surgery, salvage treatment can be successful in the case of unresectable recurrence. All patients were originally planned for adjuvant RT or CRT after surgery. However, after detection of recurrence from CT simulation, these plans changed and patients were treated with a course of definitive salvage CRT. Treatment intensification may be able to salvage a select group of patients.

With improved resolution of computed tomographic with i.v. contrast and the possibility for aggressive recurrence in patients, this raises the question about whether radiologists should also be reading CT simulation scans to check for disease recurrence during the period after surgical resection and before the initiation of adjuvant radiotherapy. It may be possible that recurrences that can be seen on CT simulations may be missed and early opportunities for detection are not 
Table I. Patient characteristics.

\begin{tabular}{|c|c|c|c|c|c|c|c|c|c|c|}
\hline Case & $\begin{array}{l}\text { Age, years/ } \\
\text { gender }\end{array}$ & Smoker & Stage & TNM & Primary site & Margins & PNI & LVSI & Nodes, ECE & $\begin{array}{l}\text { Time to recurrence detection } \\
\text { after initial resection }\end{array}$ \\
\hline 1 & $32 \mathrm{M}$ & 0 py & IVA & T2N2M0 & Right tongue & - & - & + & $2 / 36,+$ in 1 node & 4 Weeks \\
\hline 2 & $52 \mathrm{~F}$ & 30 py & III & T3N1M0 & Larynx & + & - & - & $1 / 74,-$ & 6 Weeks \\
\hline 3 & $58 \mathrm{M}$ & 25 py & IVA & T4aNOM0 & Oral cavity & - & + & - & $0,-$ & 6 Weeks \\
\hline 4 & $55 \mathrm{M}$ & 30 py & IVA & T2N2bM0 & Right tongue & - & + & - & $2 / 67,-$ & 5 Weeks \\
\hline
\end{tabular}

M: Male; F: female; TNM: tumor, nodal and metastases staging; py: pack-years; PNI: perineural invasion; LVSI: lymphovascular space invasion; ECE: extracapsular extension. All patients were human papillomavirus-negative.

Table II. Planned treatment compared with actual salvage treatment received.

\begin{tabular}{lccc}
\hline Case & Original treatment plan & Salvage radiotherapy & Salvage chemotherapy \\
\hline 1 & 66 Gy with concurrent cisplatin & $70 \mathrm{~Gy}$ & Concurrent cisplatin \\
2 & $66 \mathrm{~Gy}$ with concurrent cisplatin & $70 \mathrm{~Gy}$ & Concurrent cisplatin \\
3 & 60 Gy only & $70 \mathrm{~Gy}$ & Concurrent cisplatin; cisplatin, erbitux, 5-fluorouracil after progression \\
4 & $66 \mathrm{~Gy}$ with concurrent cisplatin & $70 \mathrm{~Gy}$ & Concurrent cisplatin \\
\hline
\end{tabular}

being capitalized upon to ensure the most subsequent salvage treatment options.

Overall, three out of four patients are currently alive with a median follow-up time of 12 months. One patient did secondary to pulmonary embolism one month after completion of salvage therapy. Two surviving individuals have had no major complaints and are doing well with no evidence of disease. Another patient, while alive, has a poor prognosis due to an aggressive local recurrence of buccal tumor, and is currently receiving palliative chemotherapy at our Institution. His prognosis and outcome is consistent with the literature (8), which suggests that buccal tumors are very aggressive and have poor outcomes. Overall, none of the patients included in this series have the best prognosis, especially taking into consideration their aggressively occurring disease and their negative human papillomavirus status (9). Despite this, the timely initiation of salvage therapy does allow for prolonged survival.

In conclusion, salvage of patients with recurrent disease prior to completion of adjuvant therapy is possible with a transition from adjuvant to definitive CRT. The use of concurrent cisplatin along with intensity-modulated RT as salvage treatment appears to be an option for patients in whom further surgery is not possible.

\section{References}

1 Tschudi D, Stoeckli S and Schmid S: Quality of life after different treatment modalities for carcinoma of the oropharynx. Laryngoscope 113: 1949-1954, 2003.
2 Cooper JS, Pajak TF, Forastiere AA, Jacobs J, Campbell BH, Saxman SB, Kish JA, Kim HE, Cmelak AJ, Rotman M, Machtay M, Ensley JF, Chao KSC, Schultz CJ, Lee N and Fu KK: Postoperative concurrent radiotherapy and chemotherapy for high-risk squamous-cell carcinoma of the head and neck. N Engl J Med 350: 1937-1944, 2004.

3 Udoff RA, Elam JC and Gourin CG: Primary surgery for oropharyngeal cancer. Otolaryngol Head Neck Surg 143: 644$649,2010$.

4 Villa A and Sonis S: Toxicities associated with head and neck cancer treatment and oncology-related clinical trials. Current Problems in Cancer http://dx.doi.org/10.1016/j.currproblcancer. 2016.06.0012016.

5 Bossi $\mathrm{P}$ and Alfieri S: The benefit of a multidisciplinary approach to the patient treated with (chemo) radiation for head and neck cancer. Curr Treat Options Oncol 17: 1-10, 2016.

6 Bensadoun RJ, Benezery K, Dassonville O, Magne $\mathrm{N}$, Poissonnet G, Ramaioli A, Lemanski C, Bourdin S, Tortochaux J, Peyrade F, Marcy PY, Chamorey E, Vallicioni J, Seng H, Alzieu C, Gery B, Chauvel P, Schneider M, Santini J, Demard $\mathrm{F}$ and Calais G: French multicenter phase III randomized study testing concurrent twice-a-day radiotherapy and cisplatin/5fluorouracil chemotherapy $(\mathrm{BiRCF})$ in unresectable pharyngeal carcinoma: Results at 2 years (FNCLCC-GORTEC). Int J Radiat Oncol Biol Phys 64: 983-994, 2006.

7 Egloff AM, Lee JW, Langer CJ, Quon H, Vaezi A, Grandis JR, Seethala RR, Wang L, Shin DM, Argiris A, Yang D, Mehra R, Ridge JA, Patel UA, Burtness BA and Forastiere AA: Phase II study of cetuximab in combination with cisplatin and radiation in unresectable, locally advanced head and neck squamous cell carcinoma: Eastern Cooperative Oncology Group trial E3303. Clin Cancer Res 20: 5041-51, 2014. 
8 El-Fol HA, Noman SA, Beheiri MG, Khalil AM and Kamel MM: Significance of post-resection tissue shrinkage on surgical margins of oral squamous cell carcinoma. J Craniomaxillofac Surg 43: 475-482, 2015.

9 Joseph AW, Guo T, Hur K, Xie Y, Yin L, Califano JA, Ha PK, Quon H, Richmon JD, Eisele DW, Agrawal N and Fakhry C:
Disease-free survival after salvage therapy for recurrent oropharyngeal squamous cell carcinoma. Head Neck 38(Suppl 1): E1501-1509, 2016.

Received September 8, 2016

Revised November 24, 2016

Accepted November 28, 2016 